\title{
Research on Line Structured Light Calibration Method
}

\author{
Wenhui Chang ${ }^{1, a}$, Li Jing ${ }^{2, b}$ \\ ${ }^{1}$ Yanjing Institute of Technology ,Yanjiao Hebei Province 065201. China \\ ${ }^{2}$ Yanjing Institute of Technology ,Yanjiao Hebei Province 065201. China \\ aemail:wenhui2915@126.com, bemail:hongtaosheji@126.com
}

Keywords: 3D scanning system, line structure light, vertical calibration plate

\begin{abstract}
During the measurement procedure of line structured light 3D scanning system, there is a key about how to calibrate the whole measuring system. In order to solve the calibration problem in the line structured light 3D measured system such as requirements for high accuracy calibration models, complicate calibration procedures and so on. This paper presented a calibration method combining the vertical calibration board with 3D scanning measurement system, this method is simple, easy to operate and the assistant adjustment equipment is unnecessary. Experiment showed that this method can attain relative accuracy about $0.51 \%$ which indicates the rationality of this method.
\end{abstract}

\section{Introduction}

At present, with the development of science and technology, the intelligent non-contact 3D measurement methods have gained wide attention, the line structured light scanning system is applying to precise measurement, based on computer vision technology with reliable, inexpressive and anti-interference. Its processing principle is the deformed of line laser stripe due to the height adjustment of the target object. Capturing the distortion of light pattern, and obtaining the dimensional information of the object surface based on the position relationship between the laser and camera coordinate system. Therefore, the 3D measurement system parameter calibration is one of the key loop of entire detection tasks.

Normally, the calibrate methods of structure sensor is drawing method, toothed target calibration method, mechanical adjustment method and so forth. The first two methods both require precise expensive auxiliary equipments, and not suitable for field calibration, besides that, the mechanical adjustment for the factors involved in too many legal, making measurement precision is lower, besides it was proposed based on the calibration reference coplanar method, but this method is to create a three-dimensional world coordinate system in the plane of reference, each time the reference position moves, the positional relationship between the world coordinate system and camera coordinate system must be readjusted [1].

In response to these problems, a vertical scale grid Othello plate is designed as a calibration target system, and improving the traditional calibration methods. Result shows that the improved method can obtain the plane equation of camera coordinate system only single measurement, and the calibration of coordinate system can also be obtained easily. This method doesn't require expensive precision equipment, simple operation and is suitable for field calibration.

\section{Parameter Calibration of Line Structured Light Scanning System}

Calibration of line structured light 3D scanning system includes two loops, first is the camera spatial location model parameter calibration and the second is the calibration of line structured light plane.

(1)Calibration of camera model parameter

Camera parameters can be obtained based on the principle of coplanar, non-coplanar and self-calibration. Accounting for this, besides taking into account the simplicity and accuracy of line structured light plane. Article designs the vertical calibration plate as shown in figure 1, and the 
corner of vertical calibration plate also can be extracted as the calibration points needed in experiment, as shown in figure 2.

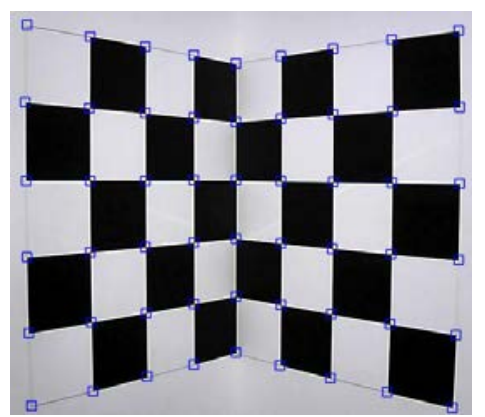

Fig.1 .Vertical calibration chessboard captured by camera

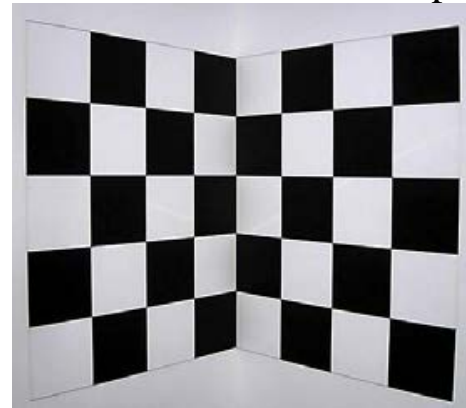

Fig.2. Corner extraction of vertical by calibration board

Tsai camera calibration based on the radial constraint and Zhang Zhengyou calibration based on planar target are mainly methods of camera calibration[2]. Considering the camera in experiment is general, only the first-order radial distortion is considered in the process of camera calibration, this paper chooses the Tsai calibration method. Firstly most of camera model parameters are solved by the radial collimator constraints, then the effective focal length, nonlinear distortion factor and other parameters are obtained so that the final completion of calibration of the camera[3,4].

(2)Parameter calibration of line structured light plane

The design of line structured light scanning system is driven by a stepping motor line lasers, cameras and a rotating platform composed of the entire scanning system platform shown in figure 3.firstly the laser projects the line structure light beam to the target, the deformation line structured light strip can be obtained and captured due to the height modulate of measured object. Among the deformation stripes the location information between line laser and camera and depth data of the measured surface is included, and the three-dimensional information of target object can be obtained based on the cross-ratio invariance principle, after scanning the whole surface using stepper motor the entire three-dimensional contour data can be obtained completely[5].

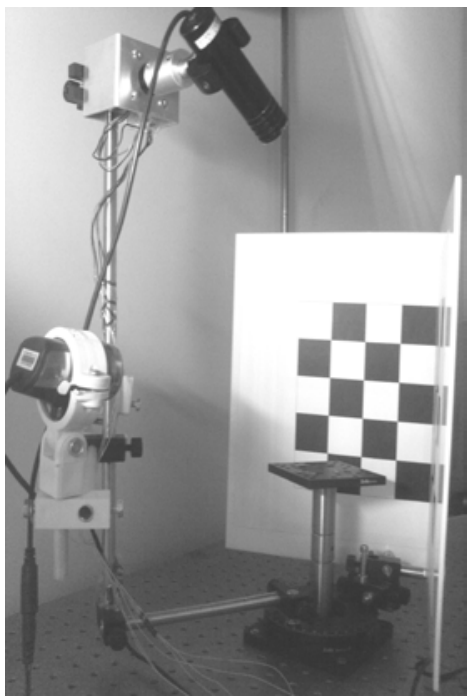

Fig. 3. 3D scanning prototype used in this experiment 


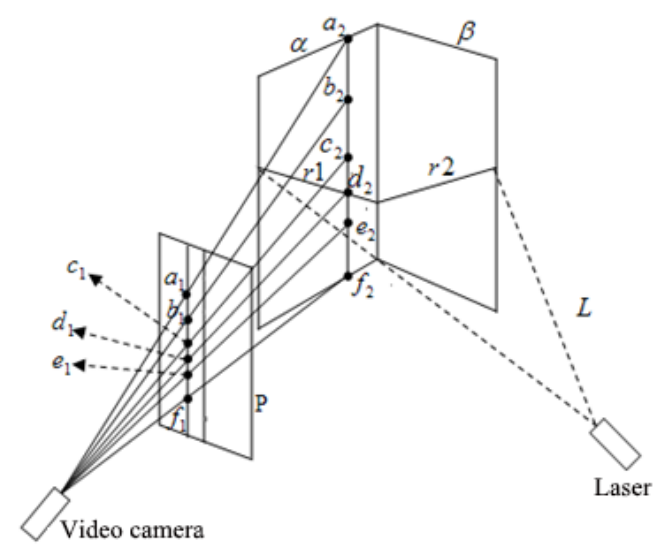

Fig.4. The principle chart of line structure light measurement

Calibration principle of line structure light 3D scanning system designed in title is shown as figure 4.where $\alpha$ and $\beta$ represent two mutually perpendicular calibration level surface, $L$ is laser plane and $P$ is image plane, $r_{1}, r_{2}$ are the intersecting lines between line structure plane and vertical calibration plane. $a_{2}, b_{2}, c_{2}, e_{2}, f_{2}$ are collinear five corners of calibration plane extracted using Harris operator, the calibration can be divided into the following steps:

1. calibrate the plane equation based on the parameters of camera. Assuming the equation of $\alpha$ in camera coordinate system is:

$$
a x_{c}+b y_{c}+c z_{c}+d=0
$$

Among $a, b, c, d$ is four unknown coefficients of $\alpha$, choosing four coordinate in world coordinate system on plane $\alpha$, translate the world coordinate into camera coordinate combining formula (2).

$$
\left[\begin{array}{l}
x_{c} \\
y_{c} \\
z_{c} \\
1
\end{array}\right]=\left[\begin{array}{cc}
R & T \\
0^{T} & 1
\end{array}\right]\left[\begin{array}{l}
x_{w} \\
y_{w} \\
z_{w} \\
1
\end{array}\right]
$$

Where the coordinate of camera coordinate system is shown as $\left[x_{c}, y_{c}, z_{c}\right]$,but the coordinate of world coordinate system is shown as $\left[x_{w}, y_{w}, z_{w}\right]$,the $a, b, c, d$ can be obtained combing any four coordinates and the following formula, then the plane equation can be achieved.

2.As the pixel coordinate and world coordinate of $a_{1}, b_{1}, c_{1}, e_{1}, f_{1}$ are given data, the cross-ratio invariance principle can be used to calibrate the pixel coordinate of points of intersection by choosing any three coordinate among $a_{1}, b_{1}, c_{1}, e_{1}, f_{1}$, then combining the internal parameters the coordinate of $d_{1}$ can be achieved. In the same way the eight other coordinate in camera coordinate system also can be obtained.

3. taking any three non-collinear coordinate $A, B, C$ among nine calibrate coordinate, assuming they are separately $A\left(x_{a}, y_{a}, z_{a}\right), B\left(x_{b}, y_{b}, z_{b}\right), C\left(x_{c}, y_{c}, z_{c}\right)$, then the line structure light plane equation can be calibrated based on the following formula.

$$
\left|\begin{array}{ccc}
x-x_{c} & y-y_{c} & z-z_{c} \\
x_{a}-x_{c} & y_{a}-y_{c} & z_{a}-z_{c} \\
x_{b}-x_{c} & y_{b}-y_{c} & z_{b}-z_{c}
\end{array}\right|=0
$$

\section{Experiment and Result analysis}

In experiment, a semiconductor laser emitter ,camera, rotating plate, vertical calibration and optical platform are used to get a line structure 3D system, where the target is placed on a fixed optical platform, the vertical calibration plate, line laser and camera are set on the rotating plate. 
In order to avoid outside noise during experiment, apart from the process of camera calibration the whole experiment is taken in the darkroom, the calibration results are shown in table 1.

Table1:Intrinsic Parameters of Camera

\begin{tabular}{c|c|l|c|c}
\hline \hline$f$ & $C_{x}$ & $C_{y}$ & $S_{x}$ & $K$ \\
\hline 3.6 & 325.35 & 268.16 & 1.011 & 0.00044708 \\
\hline \hline
\end{tabular}

After obtaining the model parameters of camera, the line structure light plane parameter can be calibrated, the captured image and the single pixel stripe image as shown in figure 5.

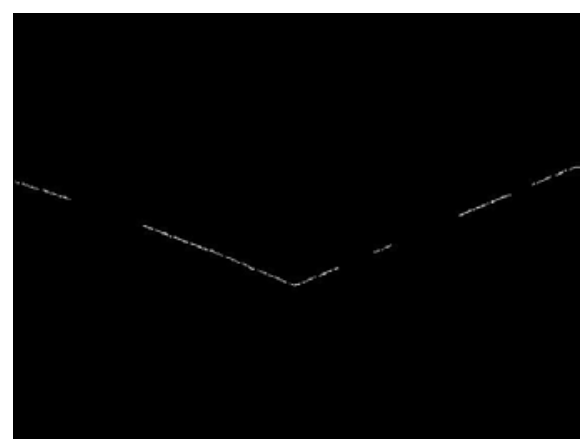

Fig.5.The light bar image and refinement in experiment

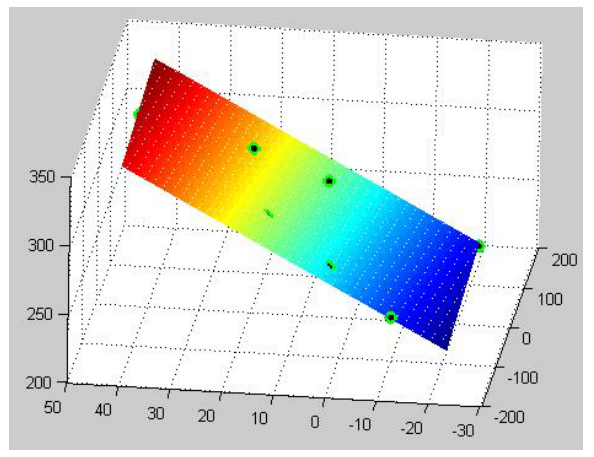

Fig.6. Line structure plane fitted by nine feature points

Where the two straight is taken between laser plane and calibration plate, and then only calculate three feature points of two straight lines can we obtain the line structure light plane equation. In this paper, detect the corners using Harris, and obtain the required calibration point, at last the light plane is calibrated combing the method of line structure light plane introduced in the front of this paper. Figure 6 shows the line structure light plane obtained through nine feature points. The equation is as shown:

$$
0.5713 x+1.9099 y+z+258.1122=0
$$

In order to determine the accuracy of the calibration method, a regular tetrahedron standard gauge block was used as the measured model, the measure result obtained is shown in table 2 .

Table2:Accuracy Analysis of System

\begin{tabular}{c|c|c|c|c}
\hline \hline Edge & Standard & Measurement value & Absolute error & Relative error \\
\hline AB & 20 & 20.10 & 0.10 & $0.50 \%$ \\
\hline AC & 20 & 20.08 & 0.08 & $0.40 \%$ \\
\hline AD & 20 & 20.17 & 0.17 & $0.85 \%$ \\
\hline BC & 20 & 20.12 & 0.12 & $0.60 \%$ \\
\hline BD & 20 & 20.04 & 0.04 & $0.20 \%$ \\
\hline CD & 20 & 19.89 & 0.11 & $0.55 \%$ \\
\hline ave & & 0.10 & $0.51 \%$ \\
\hline \hline
\end{tabular}


The data shows that the line structured light three-dimensional measurement system can achieve approximately $0.51 \%$ relative accuracy. And as can be seen from the test results that the proposed calibration method reasonable idea, principle is correct and the desired results can be achieved.

\section{Conclusion}

Taper presents a calibration method of 3D scanning system, this method obtains the feature points based on the vertical calibration plate. The coordinate of feature points can be calculated By extracting the corners of vertical calibration plate combing the cross-ratio invariant principle, by obtaining the plane equation in camera coordinate system to achieved the calibration of 3D scanning system. Finally, the article tests the method using the tetrahedron calibration standard gauge block, and the results present higher precision of the method without any auxiliary equipment, besides the convenience and fast facilitates the visual measurement calibration work greatly.

\section{References}

[1] Gao Y, Wang M, Ji R, Wu X, Dai Q, “3D object retrieval with hausdorff distance learning,”IEEE Transactions on industrial electronics 61:2088-2098.2013.

[2] Ma,Shujing, Fang Bin, "Optical image reconstruction using frequency-domain simulations: Optimization of detector positions,” Proceedings of 2012 International Conference on Modeling, Identification and Control, ICMIC 2012,pp.68-73,2012.

[3] Tsai, R.Y, “An efficient and accurate camera calibration technique for 3d machine vision,” IEEE Conf. Computer Vision and Pattern Recognition.1986, pp.364-374.Tsai, R.Y, “An efficient and accurate camera calibration technique for 3d machine vision,” IEEE Conf. Computer Vision and Pattern Recognition. (1986) 364-374.

[4] Carles Matabosch, Joaquim Salvi, David Fofi and Fabrice Meriaudeau, “A refined range image registration technique for multi-stripe laser scanner," Proceedings of SPIE-The International Society for Optical Engineering, v6070, 2006.

[5] Kim MY, Ayaz SM, Park J, Roh YJ, “Adaptive 3D shape inspection system of automotive part based on structured light pattern,”Optics and Laser Technology 43:1-8.2013. 